\title{
Archetype alignment: A two-level driven semantic matching approach to interoperability in the clinical domain
}

\author{
Damon Berry \\ Technological University Dublin, damon.berry@tudublin.ie \\ Jesus Bisbal \\ Universitat Pompeu Fabra, jesus.bisbal@upf.edu
}

Follow this and additional works at: https://arrow.tudublin.ie/teapotcon

Part of the Computer Engineering Commons, Databases and Information Systems Commons, and the Medicine and Health Sciences Commons

\section{Recommended Citation}

Bisbal, J. and Berry, D.:A two-level driven semantic matching approach to interoperability in the clinical domain.HEALTHINF:Proceedings of the International Conference on Health Informatics, pp216--229,2009

This Article is brought to you for free and open access by the tPOT: People Oriented Technology at ARROW@TU

Dublin. It has been accepted for inclusion in Conference

Papers by an authorized administrator of ARROW@TU

Dublin. For more information, please contact

arrow.admin@tudublin.ie, aisling.coyne@tudublin.ie, gerard.connolly@tudublin.ie.

Funder: Regional Government of Catalonia, Health

Information and Quality Authority of Ireland

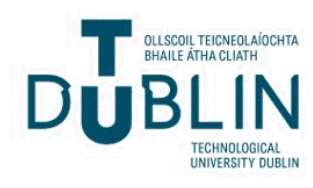




\title{
ARCHETYPE ALIGNMENT: A TWO-LEVEL DRIVEN SEMANTIC MATCHING APPROACH TO INTEROPERABILITY IN THE CLINICAL DOMAIN
}

\author{
Jesús Bisbal \\ Universitat Pompeu Fabra (CISTIB) and CIBER-BBN, Pg Circumvallacio 8, Barcelona 08003, Spain, \\ jesus.bisbal@upf.edu \\ Damon Berry \\ Dublin Institute of Technology, School of Electrical Systems Engineering, Kevin Street, Dublin 6, Ireland \\ damon.berry@dit.ie
}

Keywords: electronic health records; two-level modelling; archetypes; interoperability; semantic interoperability; ontologies; ontology alignment.

\begin{abstract}
Semantic interoperability between electronic health record systems and other information systems in the health domain implies agreement about the structure and the meaning of the information that is communicated. There are still a number of similar but different EHR system approaches. Some of the newer approaches adopt the two-layer model approach where a generic reference model is constrained by archetypes into valid clinical concepts which can be exchanged. The meaning of the concepts that are represented by an archetype can be conveyed by embedding codes from a commonly recognised terminology at appropriate points in the archetype. However, as the number of archetypes multiply it will become necessary to match archetypes from different sources to facilitate interoperability. This paper describes an approach that supports semantic interoperability between heterogeneous two-level health information systems by identifying similarities between archetypes. The approach identifies relationships between ontological terms which have been embedded in pairs of archetypes as a means of matching these terms. The matched terms can then in turn be used to identify similarities between archetypes. The limited contextual scope of an archetype simplifies this matching process.
\end{abstract}

\section{INTRODUCTION}

Safe interoperability of clinical information systems (Grimson 2001; Dick 1997) requires that the information being transferred includes all necessary context so that it can be appropriately interpreted at a site (e.g. hospital) that is different from where that information was originally captured. Misinterpretations may lead to fatal medical decisions (Kohn 2000). This requirement has widely been acknowledged in the medical domain (Grimson 2001). Also, in biomedical research (e.g. genomics), for example, capturing the context of information has also been clearly identified as an important requirement (Goble 2008), but a generic architectural solution has not emerged, as in the case of clinical applications.
The traditional software engineering approach to modelling any domain consists of creating a (possibly very large) model which contains all the information that is considered necessary for the software application at hand. The necessary information is identified by the user experts that take part in the project. This approach has repeatedly been followed in the medical domain, and it has proved rather unsatisfactory (Garde 2007). The medical domain is characterized by being (MartinezCosta 2008):

1. Large: e.g. a well known clinical taxonomy, SNOMED-CT, contains over 350.000 atomic concepts and 1.5 million relationships;

2. Complex: different views of information, requirements, and granularity, all of which must be represented in the model; and 
3. Open-ended: advances in clinical research constantly update clinical practice, which in turn change the type of information that is considered necessary for a given application.

Therefore, instances of a single-model approach necessarily represent a narrow view of the domain that needs to be represented, as understood by the specific set of users that have been involved in the requirements phase. If a different set of users is involved, it is likely that a rather different set of information items will be judged as necessary, although it will be equally valid.

Currently, it is believed that the most promising approach to model the clinical domain for interoperability purposes consists of separating what is Information, from what is domain Knowledge. Accordingly, the clinical domain is modelled using the so-called two-level modelling approach (Grimson 1998; Beale, 2002; Garde 2007). The first level, referred to as the Reference Model, contains a very reduced set of building blocks or classes of an object model, which have a very abstract meaning (i.e. Element, Item, Entry, Section, Composition, and Folder). These building blocks are organised in a hierarchical structure according to a set of generic constraints. Due to the abstract meaning of these classes, however, any clinical concept can be modelled using only this limited set of classes. A clinical concept (e.g. blood pressure, problem list) that has been modelled by applying further clinical constraints to these abstract classes represents the second level of this architectural approach, the Knowledge, and is referred to as Archetype. Archetypes are the basis on which interoperability is performed in the two-level approach. Figure 1 shows a representation of the actors involved in this approach, as well as their relationships (Beale 2002).

It must be noted that the two-level modelling approach is currently being adopted by the major medical information standardization bodies: CEN 13606 (or EHRcom, http://www.centc251.org/), and HL7 RIMv3 (http://www.hl7.org/). Additionally, the OpenEHR (http://www.openehr.org/) foundation provides another specification and open source implementation of this approach. Although attempts have been made, there is still further work to harmonise the reference models and archetype models used by these efforts.

Therefore, for the time being, in order to achieve semantic interoperability of clinical applications, the two-level modelling approach does not suffice. At the very least, a mapping between the different (de jure or de facto) standards is needed (Dogac 2005; Martinez-Costa 2008; Iakovidis 2007). However, it

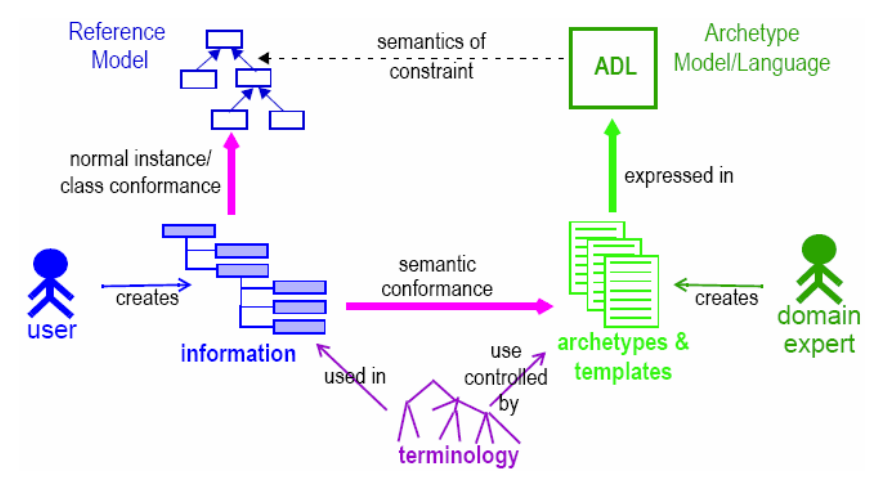

Figure 1: Two-level modelling approach (Beale 2002).

is also quite likely that more sophisticated mappings between archetypes will also be required. As described above, archetypes are the unit of communication between interoperating applications, as they define the minimum context that must be considered for safe communication. Thus archetypes must be agreed upon before communication. It does not seem feasible, however, to expect that all professionals of all disciplines will agree on exactly all details of the archetypes associated to the data they would like to exchange. If this approach becomes widely accepted, it is certain that the number of available archetypes will become very large (at the time of writing there are aproximately 700 archetypes in OpenEHR). Although archetypes are annotated with terms from standardised ontologies (terminologies, taxonomies, etc), there will still exist differences both at the archetype level, as well as at the terminology level. Local variations at the archetype level will stem from specialization of archetypes for specific purposes and research projects, and due to competing standards. Also, there are several widely used terminologies that could be used to annotate archetypes (e.g. SNOMED-CT, MeSH, NCI, FMA). Local ontologies are also used to annotate archetypes. Therefore, a sound and general process for matching archetypes is essential.

This paper describes a novel approach to address semantic interoperability in the healthcare domain. Archetypes are used to scope the context of the matching process that will allow two independent healthcare providers to interoperate. It structures the matching algorithms at two different levels, the terminology level and the archetype level, leveraging the most mature research on ontology matching. The context of archetype will limit the matching space, to allow for more accurate mapping results and, due to the nature of archetypes, ultimately to very high level of automation. 


\section{RELATED WORK}

The two-level modelling paradigm was originally proposed by the EU-funded project Synapses (Grimson 1998). Since then, it has evolved (Beale 2002) incorporating additional constraints. This evolution has not produced a unified approach, but there are three major players. ISO 13606 and HL7's CDA RIMv3 are EU and USA standards. OpenEHR is promoted by a commercial company (http://oceaninformatics.biz), and is having a significant impact. Some research projects aim at facilitating the interoperability between these approaches (Iakovidis 2007).

The Artemis project developed a framework to map archetypes between different standards (Dogac 2005). It defined a syntactic transformation of (ADL-defined) archetypes into OWL format. The project developed a tool called OWLmt that was used to manually define the mappings between the archetypes, and then automatically map data instances conforming to the source archetype into instances conforming to the target archetypes. The present paper aims at avoiding this manual mapping.

The Poseacle project (Martinez-Costa, 2008) is taking a software engineering approach for the semantic transformation of ADL archetypes into OWL. The final goal is the transformation of one archetype expressed in one standard into the same archetype expressed into a different standard. In contrast, the work presented here aims at aligning two archetypes (expressed in same or different standards) that may define similar but not necessarily the same concepts.

An ontology is the explicit conceptualization of a domain agreed upon by a community of users (Martinez-Costa 2008). Due to the large number of ontologies currently available for many domains and applications, there is a need to match different ontologies (i.e. to find equivalences) or to align them (when the domains only partially overlap). A large body of research exists on ontology matching and ontology alignment approaches (Tan 2007). There are two main categories of ontologies (Garde 2007). 'Ontologies of reality' describe real phenomena, while 'ontologies of information' contain the information models of the content to be stored or communicated. A collection of Archetypes is an information ontology. The ultimate goal of the research presented here is to adapt and extend existing ontology alignment techniques to the particular case of archetypes, in order to achieve better quality results and more automation in semantic interoperability.

\section{SYSTEM ARCHITECTURE}

As archetypes are the artefacts around which interoperability is built, there is a need to automatically map between archetype definitions. The architecture of the system being implemented to achieve this goal is shown in Figure 2. The architecture is clearly separated in two levels. Archetypes (e.g. blood cholesterol) are used to guide the alignment process and are used at both levels.

As described in the previous section, the concepts defined in archetypes using classes from the reference models are normally annotated using terms from standard terminologies (e.g. MeSH). These terminologies must be harmonized before archetypes can be aligned. However, the alignment of such ontologies is a significant task by itself. Thus, a semantic interoperability approach based on manipulating large terminologies (ontologies) would suffer scalability (and quality) problems. It should be noted, however, that archetypes provide a better context for alignments than general. It is easier to create mappings if the ontologies to map are smaller and scoped to the application (Noy 2004). For this reason, at the upper half of Figure 2, the references to standard terminologies included in the archetypes (their 'annotations') are extracted.

These references are used to query the standard terminologies and consistently extract related concepts (Noy 2004; Jiménez-Ruiz 2007). The resulting subontologies should be of a reasonable size, since an archetype must have a limited scope. Therefore, existing alignment techniques can be applied to these subontologies (Tan 2007). The result of this step is a set of alignments between a few concepts of these standard terminologies. In some cases there already exists a metathesaurus (e.g. UMLS) which defines equivalences between terms. However, in general, especially in research environments, this will not be the case.

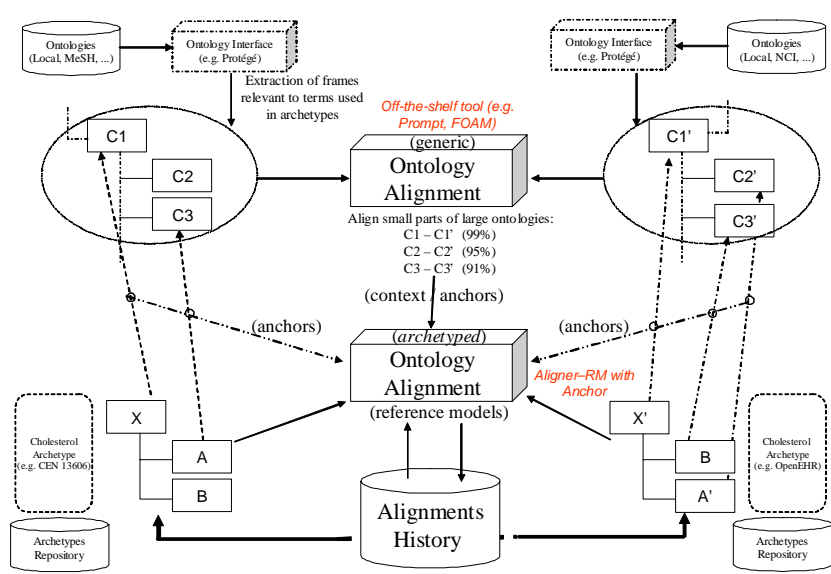

Figure 2: System architecture. 
Once this initial step has been performed, the aligned terms (concepts) are used as anchors (Noy 2001) to the next alignment module, at the lower half of Figure 2. Anchors are pairs of already aligned concepts, and are used to tune the similarity measures of neighbouring concepts, ultimately providing better quality alignments. This module will match the remaining parts of the archetypes and could be implemented using simply existing ontology alignment algorithms. However, such an approach would ignore the additional knowledge provided by the reference models upon which archetypes are built. Thus, the objective is to modify the way alignment algorithms work so that they take into account the specific characteristics of the reference models (see Section 4). Once this is done, this new module (termed in the figure Aligner-RM with Anchors), would map the archetypes. Such a mapping approach would fall into what is currently referred to as Semantic Matching (Giunchiglia 2007). To the best of the authors' knowledge, this approach has not been yet investigated in the context of archetypes, using the specific semantics of their underlying reference models and hierarchical structure (tree) of archetype definitions.

The last component of the architecture shown in Figure 2 refers to the history of alignments between archetypes successfully performed in the past. Many existing tools store previous alignments so that they may be reused in future alignment tasks (Tan 2007). However, this is always done in a local and proprietary way. The very nature of archetypes implies that they must (to a large extend) be agreed upon by a community. Also, the number of different archetypes that two different healthcare providers will be interested in exchanging (aligning) will also be limited. Finally, there are currently only three competing archetype models. Considering all these characteristics, it will be very likely that the same or very similar alignment tasks are repeatedly performed by the communities using the two-level approach. Therefore, the history of previous alignments is expected to play a very central role in an archetype-oriented alignment architecture. Given the limited scope of archetypes and the limited number of possible alignments, in the long run this history of alignments should increase the level of automation that could be achieved.

\section{IMPLEMENTATION}

The architecture described in the previous section is currently under development. It has been designed so that most of its components can be built using existing open source code publicly available from recent and current research projects. This approach reduces the overall development time and increases the quality of the resulting software, as its components are updated by the advances of these research projects.

All ontological components (terminologies and archetypes) are used in their OWL format. Although OWL is not yet the only language to describe ontologies, it is becoming widely accepted. That being said, existing terminologies, taxonomies and ontologies have been written in many different languages. For example, MeSH and NCI have their own proprietary formalisms (now commonly expressed also in XML). Biological ontologies are commonly expressed in OBO. Finally, archetypes have traditionally been expressed with ADL (archetype definition language).

However, the current trend is towards expressing all ontologies in OWL and, without loss of generality, this is the assumption taken for this architecture. Particularly, NCI is already available in OWL format (NCI, 2008), and MeSH can readily be transformed into OWL (Assem 2004). Finally, recent developments (Martinez-Costa 2008) argue for archetypes to be expressed in OWL, and some example archetypes exist that have already been semantically mapped into OWL. All of these contributions are being incorporated into the implementation of the architecture presented here.

Standard terminologies are too large to be managed in a single file. Thus the implementation reported here is storing and manipulating them in an OWL database, using Protégé (protege.stanford.edu) ontology management system.

The extraction of subontologies from these standard terminologies is being implemented using two of Protégé plug-ins that have this specific purpose, namely Prompt (Noy 2004) and OntoPath (Jiménez-Ruiz 2007).

The ontology alignment module shown at the upper half of Figure 2 is generic; it does not add any benefits to specialize its behaviour to the characteristics of the two-level model. Its goal is simply to align the (small) subontologies extracted from large standard terminologies. Several possible open source modules can be reused here. In particular FOAM (Ehrig 2005) is being used for the current implementation.

The most innovative contributions of the architecture being implemented are, on the one hand, the use of two levels of alignments and, on the other, the actual mapping of archetypes (module at the 
lower half Figure 2). The development of this module is the only significant implementation effort needed in order to fully realize and evaluate the architecture advocated in this paper. Its implementation will modify existing open source alignment algorithms (particularly, FOAM). Much research will be needed in order to fully exploit the specific characteristics of archetypes and reference models in the implementation of this module. The current considerations that are being taken into account, thanks to the existence of an underlying reference model, include:

1. Elements of archetypes define value ranges that the conforming data must satisfy. If these do not match in both archetypes (intersect, include), it is possible that the two archetypes may not be aligned.

2. Archetypes can also express the units in which measurements are taken. If units are different in both archetypes, interoperation is jeopardized.

3. The reference models include the concept of 'certainty'. If terms in archetypes do not satisfy certainty thresholds, automated interoperation may not be possible.

4. The building classes of the reference models in all (three) competing standards of the two-level modeling approach have abstract semantics. Although the names of the classes are not shared between these standards, their semantics are quite similar. If alignments are to be performed between archetypes of different standards, aligning algorithms based on string similarity measures will fail in this case, as class names can be very disparate. Dictionary-based approaches will not be of much help either, as all names are quite abstract. Model Management research (Atzeni 2008), see section 5, should be used to address this issue.

5. Archetypes define a hierarchical organization of classes from the reference model. The particular location of a given element of an archetype inside this hierarchy defines its context and restrains its extract meaning. Semantic matching research (Giunchiglia 2007) is being inspired by the same observations, and it will be specialized for the case of archetypes.

The evaluation of the current implementation of the architecture described in Section 3 must show the benefits of the two-level approach to alignment. It must compare the performance and quality of algorithms that do not include knowledge of reference models. FOAM is being used as the baseline for comparison purposes. In the evaluation, archetypes expressed in OWL format (particularly, cholesterol archetypes are used for the time being, taken from (klt.inf.um.es/ poseacle/ontologies.html) have been introduced as input to FOAM. This tool output a set of alignments (510, if threshold 90\%), both with concepts extracted from the archetypes as well as from the underlying reference models. Similarity measures in FOAM do not take into account the semantics of the abstract classes that build up the reference models or the constraints enforced when defining archetypes, like for example those outlined above.

\section{FUTURE WORK}

This paper has presented an architecture to facilitate the interoperability between clinical information systems that use the so-called two-level modelling. Archetypes in this modelling paradigm are seen as the centre of the interoperation process. By automatically identifying appropriate mappings between archetypes, semantic interoperability is greatly facilitated.

This architecture leverages and specializes results from on-going research projects on ontology alignment and management, and using the opensource software these produce.

Future research will be mainly focused on two areas: (sub)ontology extraction, and archetype-aware alignment algorithms. Subontology extraction can be compared to defining a view from a database. However, due to the richer semantics defined by an ontology, it must be decided how such a view is extracted, so that the resulting ontology can be considered consistent. Existing approaches (Noy 2004; Jiménez-Ruiz 2007) are based on the definition of the path traversals which identify the nodes are to be extracted. For archetypes, the nodes to be extracted are those terms used in order to annotate the concepts of an archetype.

Regarding archetype-aware alignments, Section 4 has illustrated a few examples of how this will be implemented. The reference model provides a very specific source of domain knowledge used to align archetypes. Particularly, when aligning archetypes which were built according to different reference models, there is also a need to map between these reference models, in addition to between the archetypes. It should be noted that reference models are considered by the communities of users as being reasonably stable. Also, they are not unmanageably large (by design). Therefore, the best approach to map between reference models is to leverage on a databases research field called model management 
(Atzeni 2008). In model management, when two models need to be mapped to each other (e.g. from relational to object-oriented), the constructs (e.g. table, class) of each model are mapped onto the set of constructs of a more abstract model, the supermodel. A set of transformations is applied, within the supermodel, to the set of constructs that originated from the source model, in order to transform them into constructs that can be mapped into the target model. At the end, the resulting constructs are mapped from the supermodel back into those of the target model. Such an approach is highly flexible as of the set of models and constructs that it can handle. Changes in the models do not require changes in the applications that perform those mappings. Such an approach has not been applied to map between reference models. (Martínez-Costa 2008) followed similar ideas but using software engineering principles instead of using database techniques.

Finally, as outlined in Section 3, the results history of previous alignment tasks should also be used to improve the quality and the automation of future alignments. Given the current trend (Chung 2007) towards cooperation between communities of users with similar interests, and given the community-orientated nature of archetypes, it is clear that the alignment history between archetypes should be a resource of such a community. It will be investigated how alignments between archetypes developed and used on several sites could be shared and reused by other sites. These alignments represent the understanding of all these archetypes (ontologies), and their equivalences, from the point of view of the different researchers involved in each of these mappings. This cumulative knowledge will be useful when new alignments are to be performed.

\section{ACKNOWLEDGEMENTS}

This research has jointly been funded by the Ramon y Cajal and the Jose Castillejo (JC2007-00050) programmes of the Spanish Ministry for Science and Innovation, and the EHRland project funded by Irish Health Information and Quality Authority.

\section{REFERENCES}

M. van Assem, M. R. Menken, G. et al. 'A Method for Converting Thesauri to RDF/OWL', The Semantic Web 2004, LNCS 3298, pages 17-31.
P. Atzeni, P. Cappellari, et al. 'Model-independent schema translation', VLDB Journal 17:1347-1370, Nov. 2008.

T. Beale. 'Archetypes: Constraint-based domain models for future-proof information systems', $11^{\text {th }}$ OOPSLA Workshop Behavioral Semantics, pp. 16-32, 2002.

V. Bicer, A. Dogac, et al. 'Archetype-Based Semantic Interoperability of Web Service Messages in the Health Care Domain', Journal Semantic Web 1, 2005.

K. Chung, L. Hossain, and J. Davis. 'Individual performance in knowledge intensive work through social networks', 2007 ACM SIGMIS CPR conference on Computer personnel research, pp 159 - 167.

R.S. Dick, et al., (Eds). 'The Computer-Based Patient Record: An Essential Technology for Health Care', Institute of Medicine, Revised Edition, 1997.

M. Ehrig, and Y. Sure. 'FOAM - Framework for Ontology Alignment and Mapping', in the Workshop on Integrating Ontologies, 156, pp. 72-76, October 2005.

S. Garde, E. Hovenga, et al. 'Expressing Clinical Data Sets with openEHR Archetypes: A Solid Basis for Ubiquitous Computing', International Journal of Medical Informatics. 76(3): 334-341, 2007.

C. Goble, and R. Stevens. 'State of the nation in data integration for bioinformatics', Journal of Biomedical Informatics, 41(5):687-693, October 2008.

J. Grimson. 'Delivering the electronic healthcare record for the 21st century'. Int. J. Med. Inf. 2001; 111-127.

J. Grimson, W. Grimson, et al. 'A CORBA-based integration of distributed electronic healthcare records using the Synapses approach'. IEEE Trans. Inf. Tech. in Biomedicine, 2(3):124-138, 1998.

F. Giunchiglia, M. Yatskevich, and P. Shvaiko. 'Semantic Matching: Algorithms and Implementation', Journal on Data Semantics, LNCS 4601, pages 1-38, 2007.

I. Iakovidis, A. Dogac, et al. 'Interoperability of eHealth Systems - Selection of Recent EU's Research Programme Developments', Proc. Int. Conf. eHealth: Combining Health Telematics, Telemedicine, Biomedical Eng. and Bioinformat. to the Edge, 2007.

E. Jiménez-Ruiz, et al. 'OntoPath: A Language for Retrieving Ontology Fragments', LNCS 4803, pp 897914, 2007.

L.T. Kohn,et al. (Ed.). 'To err is human: Building a safer health system', National Academic Press, 2000

C. Martínez-Costa, et al. 'A Model-driven Approach for Representing Clinical Archetypes for Semantic Web Environments'. Journal Biomedical Informatics 2008.

NCI Thesaurus, accessed July 2008 at http://ncicb.nci.nih.gov/download/evsportal.jsp

N.F. Noy, and M.A. Musen. 'Anchor-PROMPT: Using non-local context for semantic matching', in the workshop on Ontologies and Information Sharing at the International Joint Conference on Artificial Intelligence, pp 63-70, 2001.

N.F. Noy, and M.A. Musen. 'Specifying Ontology Views by Traversal', the Semantic Web Conference - ISWC 2004, LNCS 3298, pp 713-725.

H.Tan and P. Lambrix, 'A method for Recommending Ontology Alignment Strategies', the Semantic Web Conference. LNCS 4825, pages 494-507, 2007. 